\title{
V2X communications with an image sensor
}

\author{
Takaya Yamazato* \\ Nagoya University, Nagoya 464-8603, Japan \\ *Corresponding author, Email: yamazato@nagoya-u.jp
}

\begin{abstract}
This paper introduces infrastructure-to-vehicle and vehicle-to-vehicle communications using VLC. A VLC coupled with a high-speed image sensor is introduced (i.e., image sensor communication). The high-speed image sensors provide eyes for autonomous and connected vehicles. VLC imparts data reception capability to image sensors with necessary functions, which can then be provided to autonomous and connected vehicles. In this paper, some of our research on coupling VLC to high-speed image sensors is introduced, including our key findings: the basics of ISC, a vehicle motion model, and range estimation.

Keywords: VLC (Visible Light Communication), ISC (Image Sensor Communication), high-speed image sensor, OCI (Optical Communication Image Sensor), vehicle motion model, range estimation, POC (PhaseOnly Correlation)
\end{abstract}

Citation: T. Yamazato. V2X communications with an image sensor [J]. Journal of communications and information networks, 2017, 2(4): 65-74.

\section{Introduction}

Vehicle automation technology is receiving increased attention. Auto manufacturers are in a rush for an initial launch of driverless cars in the $2020 \mathrm{~s}^{[1]}$.

Autonomous vehicles operate in isolation from other vehicles by using internal sensors ${ }^{[2]}$, whereas, connected or cooperative vehicles use sensor information gathered from nearby cars and infrastructure using V2V (Vehicle-to-Vehicle) and I2V (Infrastructure-to-Vehicle) communications ${ }^{[3]}$.

Among the internal vehicle sensors that support autonomous driving, LIDAR (Light Detection and Ranging $)^{[4,5]}$ and cameras ${ }^{[6,7]}$ are the key vision sensors. The camera (i.e., an image sensor) provides "eyes" of autonomous and connected vehicles, which are used for several automotive applications based on image-processing technologies. Image sensors are being continuously installed in vehicles, and they are already indispensable devices. Major safety applications, such as pedestrian detection and lane detection, rely upon the image sensors.

Notably, image sensors can receive VLC signals. As shown in Fig. 1, data transmitted from LED traffic lights, signage, pavement markers, headlights, and tail lights can be received using the image sensor ${ }^{[8-23]}$. An LED traffic light transmits safety information to cars. LED taillights and headlights can transmit internal vehicle data to nearby cars and roadside stations. As mentioned, positioning and detection of objects are the primary application of the image sensors; they can detect their positions and data reception simultaneously. Further, LED pavement markers transmit ID information, providing precise localization or anchor data.

A notable feature of image sensors is the spatial separation of objects, as shown in Fig. 2. Thanks to the spatial separation ability, the receiver can

Manuscript received Jun. 08, 2017; accepted Aug. 09, 2017

This work is supported in part by Japan Society for the Promotion of Science (JSPS) KAKENHI (No. 16H04364). 
focus only on the meaningful pixels struck by the LEDs and throw away the rest of the pixels that may contain some noise sources. We also note that the SNR (Signal-to-Noise Ratio) is rather high even in outdoors ${ }^{[14]}$. Another interesting feature of image sensors is that the stable SNR is confirmed as long as the projected image of the LED transmitter occupies several pixels ${ }^{[19]}$. In other words, the received SNR remains stable for a certain communication distance.

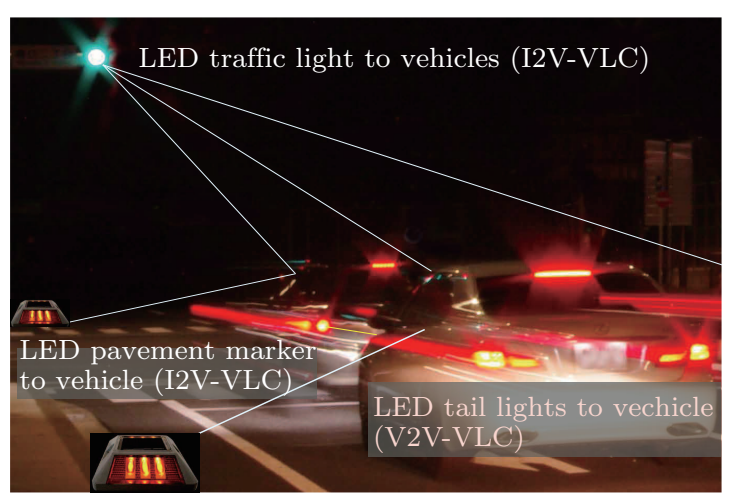

Figure 1 V2X visible light communication (Here, V2X refers to as a Vehicle-to-Everything communication, in particular V2I (Vehicle-to-Infrastructure), V2V (Vehicle-toVehicle), V2P (Vehicle-to-Pedestrian), and V2D (Vehicle-toDevice) communication, and vice versa in communication direction.)

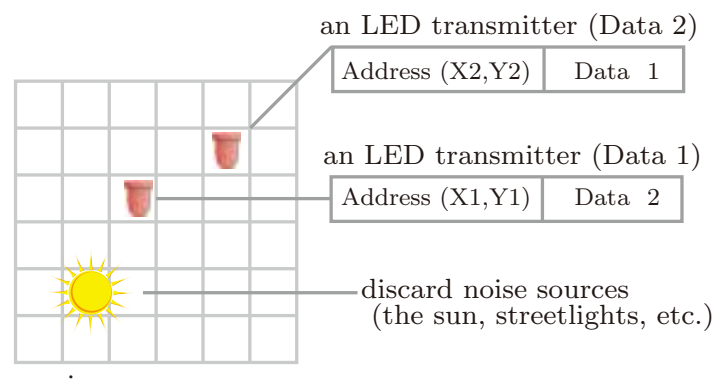

image sensor

Figure 2 Advantage of using an image sensor as the VLC receiver (The receiver selects only pixels that contains VLC signal and discards the others.)

Note that most image sensors are designed per human eye characteristics. That is, the frame rate is generally limited to $30 \mathrm{fps}$ (Frames-per-Second), or several multiples of $30 \mathrm{fps}$. This rate is too slow for visible light communication. Therefore, a frame rate of $1000 \mathrm{fps}$ or more is desirable for an image sensorreceiver.
We researched VLC using image sensors for automotive applications (i.e., ISC), mainly targeting V2X communications ${ }^{[9-23]}$. In this paper, a selection of our research on ISC is introduced including our most significant findings (e.g., the basics of ISC, a vehicle motion model for ISC, and range estimation positioning).

Two ISC receivers are introduced: a highspeed image sensor, which we have been using for years; and an OCI (Optical Communication Image) sensor ${ }^{[22,24,25]}$. The rest of the paper is organized as follows.

In section 2, the ISC for V2X is introduced. An LED array is used as a transmitter, and two ISC receivers are described: a high-speed image sensor and OCI sensor ${ }^{[22,24,25]}$.

Section 3 presents a vehicle motion model for I2V-ISC. When a high-frame-rate (e.g., 1000 fps) image sensor is used as an ISC receiver, transmitter displacement in an image plane due to vehicle vibrations can be modeled by Gaussian random processes $^{[26]}$.

ISC range estimation is introduced in section 4 . A sub-pixel range estimation is introduced, which uses phase-only correlation ${ }^{[27]}$. Finally, section 5 concludes this paper.

\section{ISC for $\mathrm{V} 2 \mathrm{X}$}

As stated previously, image sensors can detect distinct multiple illuminating sources, enabling simultaneous reception of multiple LED transmitters. For example, VLC signals transmitted from LED traffic lights, signage, pavement markers, headlights, and tail lights can be simultaneously received. Additionally, road traffic information, phase status of a traffic signal, latitude, longitude, and speed data of nearby cars can also be simultaneously received. Furthermore, if these sources are synchronized and their positions are known, range estimation or positioning can be made possible.

In this section, we describe a basic system model of V2X-ISC. To simplify the discussion, we only consider I2V-ISC, as shown in Fig. 1. 

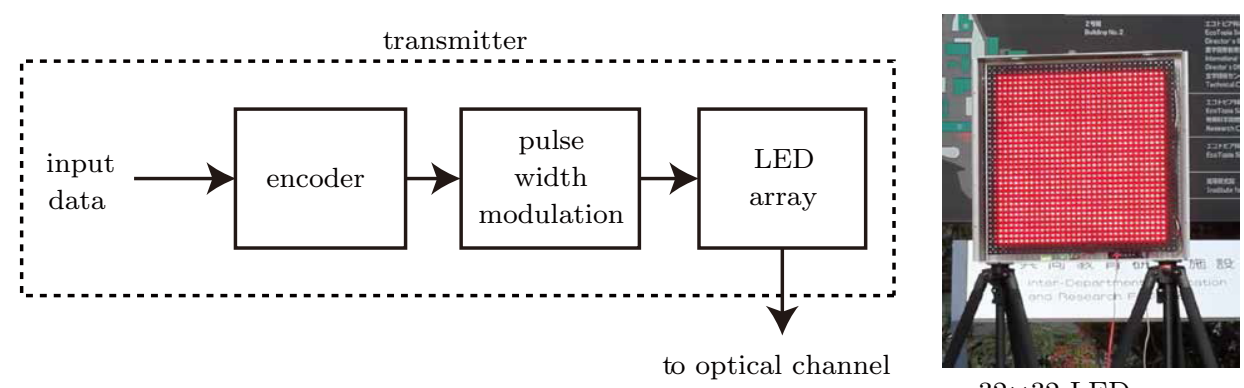

$32 \times 32$ LED array

Figure 3 An example of ISC transmitter

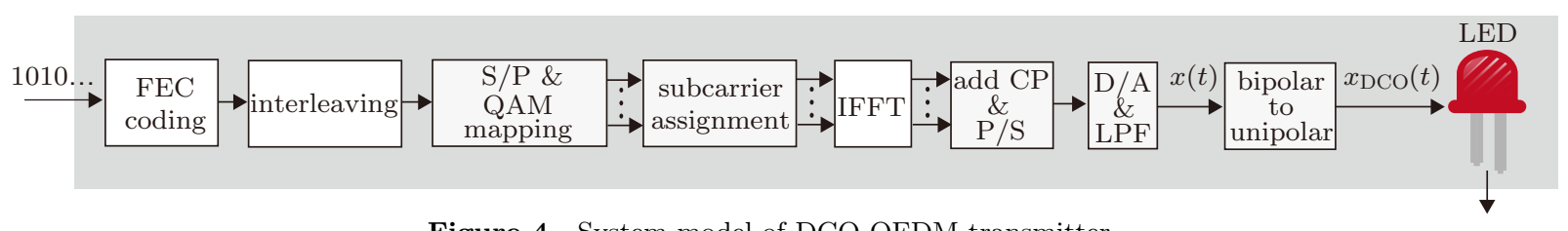

Figure 4 System model of DCO-OFDM transmitter

\subsection{Transmitter}

Any VLC transmitter can be used, but we adopt an LED array consisting of multiple LEDs arranged in a square grid. As a VLC signal is characterized as "real" and "positive", we use a simple PWM (PulseWidth Modulation) to modulate LEDs. The picture shown on the right of Fig. 3 is the $32 \times 32$ LED array we use for experiments ${ }^{[9]}$.

If very fast frame-rate image sensors or specially designed image sensors, such as OCI sensors are used, it is possible to adopt DCOOFDM (DC-biased Orthogonal Frequency Division Multiplexing) $)^{[22,24,25]}$. Fig. 4 shows the DCO-OFDM transmitter, a very common modulation scheme adopted in the literature for indoor usage.

The choice of modulation is based on available receiver bandwidth. For DCO-OFDM, a bandwidth of several $\mathrm{MHz}$ is usually required. Unfortunately, even with the ISC's frame rate at $1000 \mathrm{fps}$, the bandwidth is much narrower compared to that of OCI or a PD (Photo-Diode). Therefore, an LED array that can transmit multiple VLC signals simultaneously is usually adopted with a simple PWM.

Fig. 5 shows an example of the packet format. The header part is used for synchronization and packet identification $^{[28]}$. The data part may contain the inverted data for LED array tracking ${ }^{[9,28-30]}$.

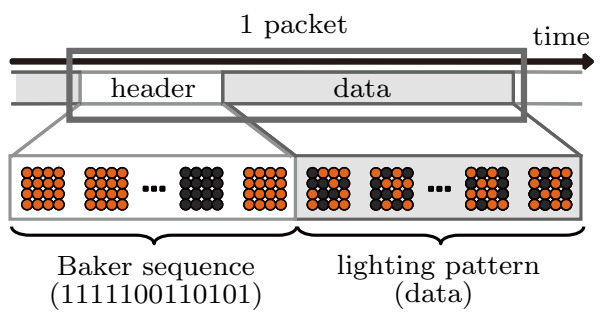

Figure 5 A packet format for an ISC (LED array transmitter is assumed, Baker sequence is adopted for synchronization purposes, and an invert lighting pattern for the tracking is adopted.)

\subsection{Receiver}

In this section, two ISC receivers are introduced: a high-speed image sensor, which we have been using for years, and an OCI sensor, developed by Takai et al. ${ }^{[22,24,25]}$.

\subsubsection{High-speed image sensor}

Fig. 6 shows the ISC receiver for the LED array transmitter ${ }^{[14]}$. The decoding process is composed of two units: an image processing unit and a VLC data decoding unit. The characteristic portion of the ISC receiver lies in the image processing unit, which detects the LED array transmitter and tracks it.

Fig. 7 shows the LED array detection. We adopt a simple subtraction of two successive frames ${ }^{[9,28]}$. Because we capture images at a rate of 1000 fps by the 
high-speed image sensor, pictures are the same except for the LEDs that are blinking. Therefore, the process removes all the background images except for the LEDs and LED array.

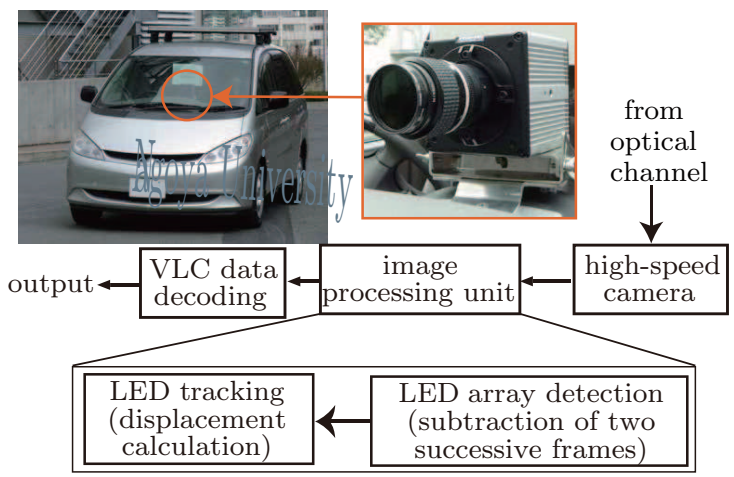

Figure 6 The block model of the high-speed image sensor receiver
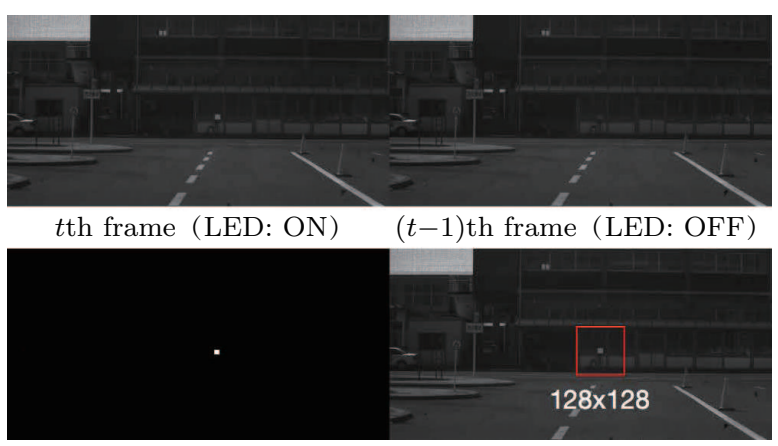

differential image

LED array detection

Figure 7 Inter-frame difference of two consecutive captured images (The LED array blinks at the rate of $500 \mathrm{~Hz}$, and the images are captured at 1000 frames/s.)

After the LED detection and tracking, we normalize the LED luminance value and then decode the data $^{[29]}$.

\subsubsection{OCI receiver}

The OCI sensor is an image sensor specially designed to achieve both image and high-speed optical communication processing in a single chip ${ }^{[14,24,25]}$. The OCI is composed of communication pixels and image pixels. The communication pixels are for the reception of optical signals, and the structure is the same as a PD. Because of this feature, the OCI can receive high-speed VLC signals and process images just like the conventional image sensors ${ }^{[14,25]}$.
Fig. 8 shows the OCI receiver for the DCO-OFDM signal. Receiver structure is almost the same as a PD receiver ${ }^{[22]}$.

\section{$2.3 \quad$ Field trials}

We place the LED array on the ground (Fig. 3), and the high-speed camera is mounted on the dashboard (Fig. 6). We drove at a speed of $30 \mathrm{~km} / \mathrm{h}$ directly toward the LED array. The communication distance is $70 \mathrm{~m}$ to $30 \mathrm{~m}$. We have achieved error-free data transmission up to $45 \mathrm{~m}$. We have also confirmed clear audio signal reception $(32 \mathrm{kbit} / \mathrm{s})^{[14]}$.

Using the OCI sensor, we achieve $55 \mathrm{Mbit} / \mathrm{s}$ transmission. Note that the transmission rate is faster than the DSRC (Dedicated Short-Range Communication $)^{[22]}$.

\section{Vehicle Motion Model for I2V-ISC}

Vehicle motion in an image plane is well modeled by a pinhole camera. This section presents a vehicle motion model for I2V-ISC. Also, we show that vehicle vibration can be modeled by the Gaussian random variables.

\subsection{Pinhole camera model}

Fig. 9 shows the projection model of the pinhole camera, where $3 \mathrm{D}$ world coordinates $(x, y, z)$ are mapped to $2 \mathrm{D}$ image coordinates $(u, v)$. The motion of the LED transmitter in the image plane $(u, v)$ for I2VISC can be expressed as follows ${ }^{[19,26,31]}$ :

$$
\lambda\left[\begin{array}{l}
u \\
v \\
1
\end{array}\right]=\left[\begin{array}{lll}
f & 0 & 0 \\
0 & f & 0 \\
0 & 0 & 1
\end{array}\right]\left[\begin{array}{ll}
\boldsymbol{R} & \boldsymbol{T}
\end{array}\right]\left[\begin{array}{c}
x \\
y \\
z \\
1
\end{array}\right],
$$

where $\lambda$ is an arbitrary scale factor, $f$ is focal length, $\boldsymbol{R}$ is a $3 \times 3$ rotation matrix indicating camera posture of the rotation matrix, and $\boldsymbol{T}$ is a translation vector indicating camera position. We considered a world coordinate component $(x, y, z)$ as the transmitter position. For I2V-ISC, the camera moves with the vehicle, while the LED transmitter remains 


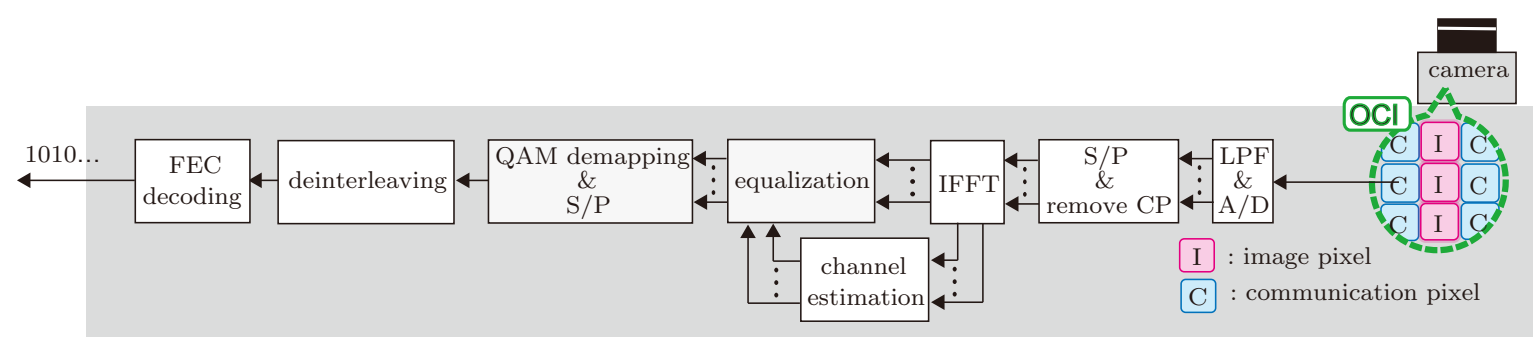

Figure 8 Receiver block model of DCO-OFDM using OCI

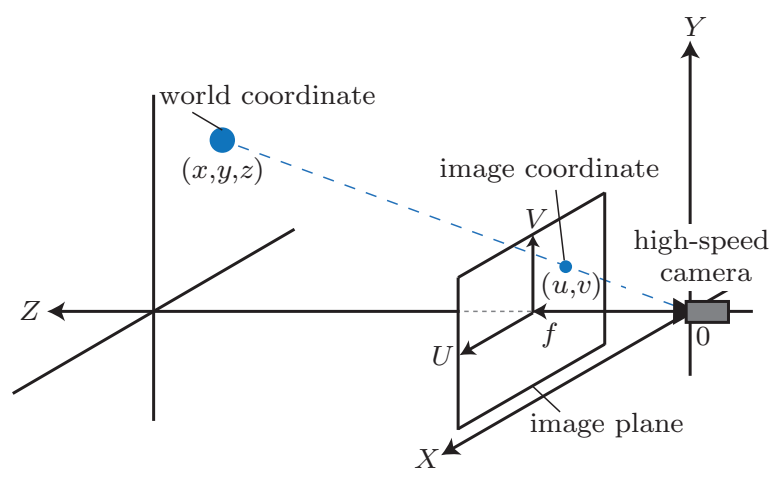

Figure 9 Pinhole camera model projecting the 3D LED transmitter coordinate $(x, y, z)$ to image coordinate $(u, v)$

static. Therefore, the camera posture $(\boldsymbol{R})$ fluctuates based on vehicle vibration, and camera position $(\boldsymbol{T})$ moves with the vehicle's movement.

\subsubsection{Displacement of the LED transmitter}

As the vehicle moves, the position of the LED source varies in the captured image plane. Displacement (exceeding one pixel) of the LED source confuses the receiver, inhibiting its ability to select the correct pixels containing data. This displacement should be addressed to develop a fast and precise tracking system $^{[19,26,31]}$.

Fig. 10 illustrates the displacement of the LED transmitter within an image plane disturbed by vehicle movement and vibrations.

We then detect the displacement by taking the difference between the transmitter positions projected onto the image plane in two consecutive frames (i.e., $\mathrm{d} u=u(t)-u(t-1)$ and $\mathrm{d} v=v(t)-v(t-1)$ between $t$ th and $(t-1)$ th frames). Characteristics of the transmitter displacement are evaluated by the probability density of the detected displacement in the horizontal $(\mathrm{d} u)$ and vertical $(\mathrm{d} v)$ directions.

The displacement is a result of the change in the rotation matrix $\boldsymbol{R}$ and the translation vector $\boldsymbol{T}$.

We define the rotation matrix $\boldsymbol{R}$ as follows:

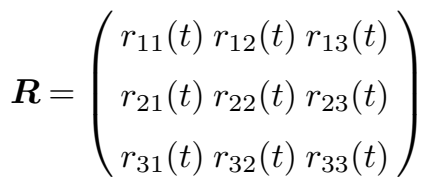

$$
\begin{aligned}
& =\left(\begin{array}{ccc}
1 & 0 & 0 \\
0 & \cos \alpha(t) & -\sin \alpha(t) \\
0 & \sin \alpha(t) & \cos \alpha(t)
\end{array}\right)\left(\begin{array}{ccc}
\cos \beta(t) & 0 & -\sin \beta(t) \\
0 & 1 & 0 \\
\sin \beta(t) & 0 & \cos \beta(t)
\end{array}\right) \\
& \times\left(\begin{array}{ccc}
\cos \gamma(t) & -\sin \gamma(t) & 0 \\
\sin \gamma(t) & \cos \gamma(t) & 0 \\
0 & 0 & 1
\end{array}\right),
\end{aligned}
$$

where $\alpha(t), \beta(t)$, and $\gamma(t)$ are the rotation angles of the $X$-, $Y$-, and $Z$-axes circumferences, respectively. Moreover, the translation vector $\boldsymbol{T}$ is

$$
\boldsymbol{T}=\left(\begin{array}{c}
T_{x}(t)+n_{x}(t) \\
T_{y}(t)+n_{y}(t) \\
T_{z}(t)+n_{z}(t)
\end{array}\right),
$$

where $T_{x}(t), T_{y}(t)$, and $T_{z}(t)$ are time functions of the vehicle's movement, and $n_{x}(t), n_{y}(t)$, and $n_{z}(t)$ represent camera displacement. 


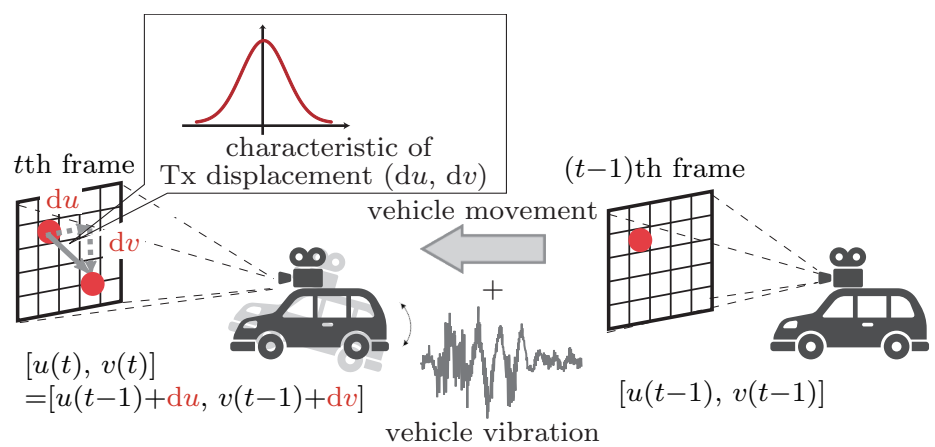

Figure 10 Displacement of the LED transmitter in an image plane owing to vehicle movement and vibrations

\subsection{Vehicle vibration components}

The vehicle vibration components can be expressed as in the following two equations:

$$
\begin{aligned}
\left(\begin{array}{l}
n_{x}(t) \\
n_{y}(t) \\
n_{z}(t)
\end{array}\right) & =\left(\begin{array}{c}
\sum A_{x f_{i}} \cos 2 \pi f_{i} t+G_{x}(t) \\
\sum A_{y f_{i}} \cos 2 \pi f_{i} t+G_{y}(t) \\
0
\end{array}\right), \\
\left(\begin{array}{c}
\alpha(t) \\
\beta(t) \\
\gamma(t)
\end{array}\right) & =\left(\begin{array}{c}
\sum A_{\alpha f_{i}} \cos 2 \pi f_{i} t+G_{\alpha}(t) \\
\sum A_{\beta f_{i}} \cos 2 \pi f_{i} t+G_{\beta}(t) \\
\sum A_{\gamma f_{i}} \cos 2 \pi f_{i} t+G_{\gamma}(t)
\end{array}\right),
\end{aligned}
$$

where $A_{x f_{i}}, A_{y f_{i}}, A_{\alpha f_{i}}, A_{\beta f_{i}}$, and $A_{\gamma f_{i}}$ are the amplitudes of the sinusoidal waveforms with frequency $f_{i} ; G_{x}(t), G_{y}(t), G_{\alpha}(t), G_{\beta}(t)$, and $G_{\gamma}(t)$ are the Gaussian random processes representing roadsurface irregularities. Note that the camera displacement in the $Z$-direction can be ignored (i.e., $n_{z}(t) \approx 0$ ), because we assume that the vehicle is driven along the $Z$-axis.

As seen in the above equation, vehicle vibrations (hereafter referred to as inherent vehicle vibrations) are induced by both the vehicle itself and roadsurface irregularities. Vibrations are transformed into an image plane, making it difficult for the ISC receiver to select correct pixels in the image plane for data reception.

Vehicle vibration frequencies are typically less than $20 \mathrm{~Hz}$, usually between $1.5 \sim 4 \mathrm{~Hz}$ and $10 \mathrm{~Hz}^{[32,33]}$. However, we can model vehicle vibration sufficient to induce displacement of an LED transmitter in an image plane using only Gaussian random processes representing road-surface irregularities. Because we are using a high-speed image sensor, the sum of the lower frequencies of 4 and 5 disappears. Only the Gaussian random processes representing road-surface irregularities remain. Consequently, Eqs. (4) and (5) can be simplified into the following equation:

$$
\left(\begin{array}{c}
n_{x}(t) \\
n_{y}(t) \\
n_{z}(t)
\end{array}\right) \approx\left(\begin{array}{c}
\tilde{G}_{x}(t) \\
\tilde{G}_{y}(t) \\
0
\end{array}\right),\left(\begin{array}{c}
\alpha(t) \\
\beta(t) \\
\gamma(t)
\end{array}\right) \approx\left(\begin{array}{c}
\tilde{G}_{\alpha}(t) \\
\tilde{G}_{\beta}(t) \\
\tilde{G}_{\gamma}(t)
\end{array}\right)
$$

where $\tilde{G}_{x}(t), \tilde{G}_{y}(t), \tilde{G}_{\alpha}(t), \tilde{G}_{\beta}(t)$, and $\tilde{G}_{\gamma}(t)$ are Gaussian random processes.

\subsection{Field trials}

We measure the actual vehicle vibration, and results show that vehicle vibrations inducing a transmitter displacement in an image plane can be modeled solely using Gaussian random processes ${ }^{[26]}$.

Our results are encouraging, particularly for LED transmitters tracking road-surface irregularities modeled by a Gaussian random process, which are the only parameters that affect transmitter displacement. Because inherent vehicle vibrations have no impact on such displacement, the transmitter displacement on an ITS-ISC depends less on the vehicle type than on road-surface conditions. In other words, we suggest that the design of a tracking system should consider the road surface rather than vehicle type. Per the obtained results, the probability of the displacement exceeding one pixel is very low $(\approx 0 \%)$, therefore displacement compensation may be unnecessary, particularly for paved-road scenarios. 


\section{ISC range estimation}

A range estimation is an exciting application of the ISC. In this section, the ISC range estimation using two LEDs whose positions are at the edge of an LED array is presented ${ }^{[16,27]}$.

Since we consider an automotive application, a challenge is how to cope with vehicle vibration.

Fig. 11 shows the block diagram of the ISC range estimation system, and Fig. 12 shows the packet format.

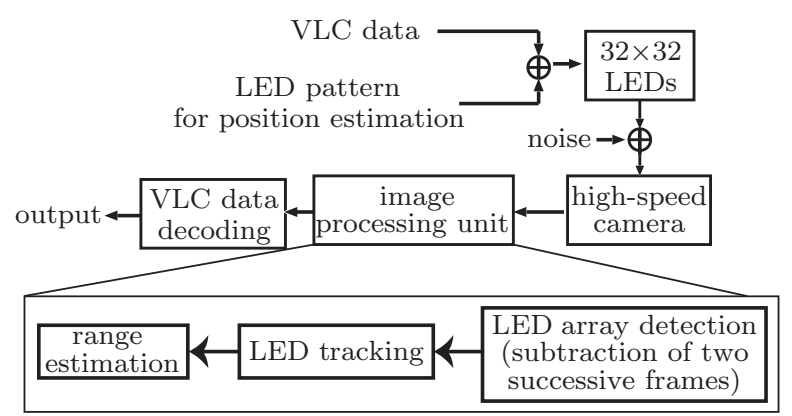

Figure 11 Block diagram of the ISC range estimation

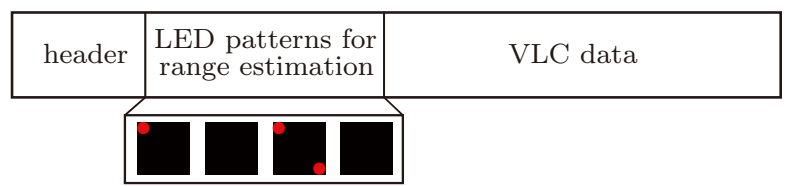

Figure 12 The packet format used for ISC range estimation

To meet the Nyquist rate, we transmit each datum twice at a rate of $1 \mathrm{~ms}^{[34]}$. We adopt an 11-bit Baker code as header data, as shown in Fig. 12. Two LED patterns for range estimation is then transmitted right after the header.

Fig. 13 shows two LED patterns and they are in the header part of the packet. We turn only the upper-left LED ON, and the others are OFF for the first LED pattern. We then turn both the upper-left LED and lower-right LED ON for the second LED pattern. The time interval between two patterns is $2 \mathrm{~ms}$.

The receiver is the same as the ISC receiver described previously, except for the range estimation unit. This means that VLC signal reception and the range estimation can be integrated.

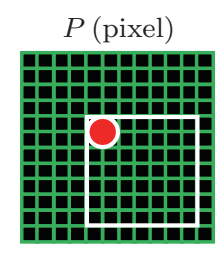

lighting pattern A

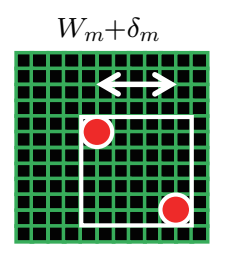

lighting pattern $\mathrm{B}$
Figure 13 Two lighting patterns seen at the image sensor

\subsection{Range estimation using POC}

We adopt a pattern-matching algorithm, well-known as POC (Phase-Only Correlation), to estimate the range in sub-pixel accuracy ${ }^{[16,19,27]}$. In POC, the amplitude components of the Fourier-transformed images are replaced with unity.

Note that using a $35 \mathrm{~mm}$ lens, the projected width of the LED array, shown in Fig. 3, is only 9 pixels at $60 \mathrm{~m}$. In this case the expected range error may be more than $3.5 \mathrm{~m}$ at a distance of $60 \mathrm{~m}$, without POC. Therefore, the POC is mandatory for precise range estimation.

Let the pixel-width of the LED array be $W_{m}$ and the pixel-height of the LED array be $W_{n}$. We assume $W_{m}$ and $W_{n}$ take pixel-value, meaning that they take some value as an integer.

Let $\delta_{m}$ and $\delta_{n}$ be some decimal values, then the actual width and the height of the LED array can be denoted as $W_{m}+\delta_{m}$ and $W_{n}+\delta_{n}$.

Please refer to Ref. [16] for the detail estimation method of $\delta_{m}$ and $\delta_{n}$ using POC. However, the method shows weakness against vehicle movement. In fact, it is reported that at most two pixels movement may occur for two consecutive frames ${ }^{[19,31]}$.

\subsection{POC range estimation and vehicle vibration}

Let us denote $f_{k}^{A}(m, n)$ as the first pattern image, and $f_{k+1}^{B}(m, n)$ be the image of the second pattern. And let $k$ be a time instant of the first image and $k+1$ be the time instant of the second image. We denote $m, n$ as the position of the image. The POC output for the inputs, $f_{k}^{A}(m, n)$ and $f_{k+1}^{B}(m, n)$, is 
given as follows.

$$
\begin{aligned}
& G_{k, k+1}(m, n)= \\
& \begin{cases}0.5, & m=V_{m}+v_{m}, n=V_{n}+v_{n}, \\
\xi_{1}(<0.5), & m=V_{m}, n=V_{n}, \\
0.5, & m=W_{m}+\delta_{m}+V_{m}+v_{m}, \\
& n=W_{n}+\delta_{n}+V_{n}+v_{n}, \\
\xi_{2}(<0.5), & m=W_{m}+V_{m}, n=W_{n}+V_{n}, \\
\epsilon, & \text { otherwise. }\end{cases}
\end{aligned}
$$

The POC peaks in pixel-level are denoted as $\xi_{1}$ and $\xi_{2}$, and their maximum values is 0.5 . The noise component is denoted by $\epsilon$. The values $V_{m}+v_{m}$ and $V_{n}+v_{n}$ are vehicle vibration components. Since $V_{m}$ and $V_{n}$ take values in pixel, they are integers. Also, $v_{m}$ and $v_{n}$ take value in sub-pixel, they are decimals.

An example of POC output is shown in Fig. 14. The peak in the middle is the POC auto-correlation output. It is the POC result of the upper left LEDs of the first pattern and the second pattern. Because of the vehicle vibration, the POC autocorrelation peak shift from the center. We denote the vehicle's vibration by $V_{m}+v_{m}$ and $V_{n}+v_{n}$.

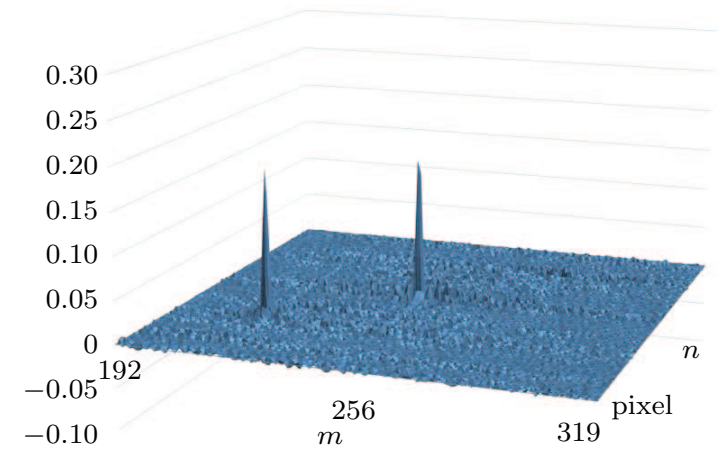

Figure 14 An example of POC output (The peak in the middle is the POC auto-correlation output. The peak offset from the origin represents vehicle vibration. The left peak is the POC cross-correlation output. The phase difference in two peaks corresponds the width and height of the LED array.)

The other peak corresponds to the POC crosscorrelation. It is the POC results of the upper-left LED and lower-right LED. The peak reflects both the vehicle's vibration, $V_{m}+v_{m}$ and $V_{n}+v_{n}$, and the pixel width $W_{m}+\delta_{m}$ and pixel height $W_{n}+\delta_{n}$ of the LED array.

\subsection{Sub-pixel POC range estimation}

As we know that the maximum value of POC output is 0.5 , shifts within a sub-pixel region correspond to the phase differences in a POC peak. So if we can estimate $\delta_{m}, \delta_{n}$, and $v_{m}$, and $v_{n}$, then the values of $\xi_{1}$ and $\xi_{2}$, become 0.5 , the maximum.

The values $\delta_{m}, \delta_{n}$, and $v_{m}$, and $v_{n}$, can be estimated by the sinc function approximation ${ }^{[16,19,27]}$.

The approximated POC output is given as

$$
G_{k, k+1}(m, n) \simeq \operatorname{sinc}\left(m+\Delta_{m}\right) \operatorname{sinc}\left(n+\Delta_{n}\right)
$$

and by varying $\Delta_{m}$ and $\Delta_{n}$, maximization can be calculated. Note that estimation of the autocorrelation POC output is denoted to simply the discussion. The peak is the one shown at the center in Fig. 14.

\subsection{Field trials}

We confirm the superior POC range estimation performance. For a $30 \sim 60 \mathrm{~m}$ range in actual driving conditions, the estimation error is fewer than $0.5 \mathrm{~m}$. The estimated pixel estimation accuracy is 0.1 pixel for this case ${ }^{[27]}$.

\section{Conclusion}

In this paper, some of our investigation regarding ISC is introduced along with our key findings, including the basics of ISC, a vehicle motion model of ISC, and range estimation (positioning). High-speed image sensors and OCI sensors are introduced. Using the OCI sensor, $55 \mathrm{Mbit} / \mathrm{s}$ transmission is possible. Note that the data rate is faster than that of DSRC. The vehicle motion model for I2V-ISC is also introduced. Section 3 presents a vehicle motion model for I2V-ISC. When a high-frame-rate (e.g., $1000 \mathrm{fps}$ ) image sensor is used as an ISC receiver, transmitter displacement in an image plane due to vehicle vibrations can be modeled by Gaussian random processes $^{[26]}$. Finally, sub-pixel range estimation using phase-only correlation is introduced, which provides good range estimation with an error of $0.5 \mathrm{~m}$ in a 30 60 m range. 


\section{References}

[1] E. Ackerman. Google's autonomous cars are smarter than ever at 700,000 miles [J]. IEEE spectrum, 2014.

[2] K. Dopart. U.S. DOT automation research program [C]//Automated Vehicle Symposium 2014, 2014.

[3] M. R. Hafner, D. Cunningham, L. Caminiti, et al. Cooperative collision avoidance at intersections: algorithms and experiments $[\mathrm{J}]$. IEEE trans. intelligent transportation systems, 2013, 14(3): 1162-1175.

[4] A. Joshi, M. R. James. Generation of accurate lane-level maps from coarse prior maps and lidar [J]. IEEE intelligent transportation systems mag., 2015, 7(1): 19-29.

[5] R. O. Chavez-Garcia, O. Aycard. Multiple sensor fusion and classification for moving object detection and tracking $[\mathrm{J}]$. IEEE trans. intelligent transportation systems, 2016, 17(2): 525-534.

[6] J. Fritsch, T. Khnl, F. Kummert, et al. Monocular road terrain detection by combining visual and spatial information $[\mathrm{J}]$. IEEE trans. intelligent transportation systems, 2014, 15(4): 1586-1596.

[7] A. Cord, N. Gimonet. Detecting unfocused raindrops in-vehicle multipurpose cameras [J]. IEEE robotics and automation mag., 2014, 21(1): 49-56.

[8] W. Huang, P. Tian, Z. Y. Xu. Design and implementation of a real-time CIM-MIMO optical camera communication system [J]. Opt. express, 2016, 24(21): 2456724579.

[9] T. Nagura, T. Yamazato, S. Arai, et al. LED array tracking method for road-to-vehicle visible light communications in the driving situation [J]. IEICE transactions on communications (Japanese edition), 2012, J95-B(2): 326-336.

[10] Y. Shiraki, T. Yamazato, H. Okada, et al. Multiple information sources recognition method for ubiquitous visible light communication using on-vehicle highspeed camera [J]. IEICE transactions on communications (Japanese edition), 2012, J95-B(11): 1517-1528.

[11] S. Nishimoto, T. Yamazato, H. Okada, et al. Overlay coding of long range data and short range data for roadto-vehicle visible light communication using led array and high-speed camera [J]. IEICE transactions on communications (Japanese edition), 2013, J96-B(2): 191201.

[12] A. Ohmura, T. Yamazato, H. Okada, et al. Accuracy improvement by phase only correlation for distance estimation scheme for visible light communications using an LED array and a high-speed camera [C]//Proceedings of the 20th World Congress on Intelligent Transport Systems, 2013.

[13] S. Usui, T. Yamazato, H. Okada, et al. A new led array acquisition method focusing on time-gradient and space-gradient values for road to vehicle visible light communication [J]. IEICE transactions on communications (Japanese edition), 2014, J97-B(7): 536-545.
[14] T. Yamazato, I. Takai, H. Okada, et al. Image-sensorbased visible light communication for automotive applications [J]. IEEE commun. mag., 2014, 52(7): 88-97.

[15] A. Ohmura, T. Yamazato, H. Okada, et al. Distance estimation scheme for integrated V2I-VLC for driving vehicle $[\mathrm{J}]$. IEICE transactions on communications (Japanese edition), 2014, J97-B(8): 695-696.

[16] T. Yamazato, S. Haruyama. Image sensor based visible light communication and its application to pose, position, and range estimations [J]. IEICE trans. commun., 2014, E97-B(9): 1759-1765.

[17] A. Ohmura, T. Yamazato, H. Okada, et al. Accuracy improvement by POC for distance estimation scheme for VLC using an LED array and a high-speed camera [J]. IEICE transactions on communications (Japanese edition), 2014, J96-B(12): 1365-1368.

[18] Y. Amano, K. Kamakura, T. Yamazato. Alamouti-type space time coding for visible light communication based on direct detection using image sensor [J]. IEICE transactions on communications (Japanese edition), 2015, J98-B(3): 307-318.

[19] T. Yamazato, M. Kinoshita, S. Arai, et al. Vehicle motion and pixel illumination modeling for image sensor based visible light communication [J]. IEEE j. sel. areas commun., 2015, 33(9): 1793-1805.

[20] K. Ebihara, K. Kamakura, T. Yamazato. Layered transmission of space-time coded signals for image-sensorbased visible light communications [J]. Journal of lightwave technology, 2015, 33(20): 4193-4206.

[21] D. Iwase, M. Kasai, T. Yendo, et al. Improving communication rate of road-to-vehicle visible light communication system using high-speed camera and LED traffic light [J]. IEICE transactions on communications (Japanese edition), 2016, J99-B(2): 97-104.

[22] Y. Goto, I. Takai, T. Yamazato, et al. A new automotive VLC system using optical communication image sensor [J]. IEEE photonics journal, 2016, 8(3): 1-17.

[23] Y. Kawai, T. Yamazato, H. Okada, et al. Reduction of resume time for vehicle positioning after occlusion using the V2I-VLC tracking method considering occlusion [J]. IEICE transactions on communications (Japanese edition), 2017, J100-B(1): 30-33.

[24] I. Takai, S. Ito, K. Yasutomi, et al. LED and CMOS image sensor based optical wireless communication system for automotive applications [J]. IEEE photonics journal, 2013, 5(5): 6801418 .

[25] I. Takai, T. Harada, M. Andoh, et al. Optical vehicleto-vehicle communication system using LED transmitter and camera receiver [J]. IEEE photonics journal, 2014, 6(5): 1-14.

[26] M. Kinoshita, T. Yamazato, H. Okada, et al. Modeling and parameter estimation of vehicle vibration inducing transmitter displacement in its image sensor communication [C]//IEEE International Conference on Communications Workshop on Optical Wireless Communi- 
cations, IEEE Communication Society, 2017.

[27] T. Yamazato, A. Ohmura, H. Okada, et al. Range estimation scheme for integrated I2V-VLC using a highspeed image sensor [C]//IEEE International Conference on Communications Workshops (ICC), 2016: 326-330.

[28] T. Nagura, T. Yamazato, M. Katayama, et al. Tracking an LED array transmitter for visible light communications in the driving situation $[\mathrm{C}] / /$ The 7 th International Symposium on Wireless Communication Systems (ISWCS), 2010: 765-769.

[29] S. Nishimoto, T. Nagura, T. Yamazato, et al. Overlay coding for road-to-vehicle visible light communication using LED array and high-speed camera [C]//The 14th International IEEE Conference on Intelligent Transportation Systems (ITSC 2011), 2011: 1704-1709.

[30] Y. Shiraki, T. Nagura, T. Yamazato, et al. Robust receiver design for road-to-vehicle communication system using LED array and high-speed camera [C]//The 18th World Congress on ITS, 2011.

[31] M. Kinoshita, T. Yamazato, H. Okada, et al. Motion modeling of mobile transmitter for image sensor based I2V-VLC, V2I-VLC, and V2V-VLC [C]//Globecom Workshops (GC Wkshps), 2014: 450-455.

[32] Y. Qiu, M. J. Griffin. Transmission of fore-aft vibration to a car seat using field tests and laboratory simulation [J]. J. sound vib., 2003, 264(1): 135-155.

[33] J. Plouzeau, D. Paillot, B. Aykent, et al. Vibrations in dynamic driving simulator: study and implementation [C]//CONFERE 2013, 2013.

[34] S. Arnon. Visible light communication [M]. Cambridge: Cambridge University Press, 2015.

\section{About the authors}

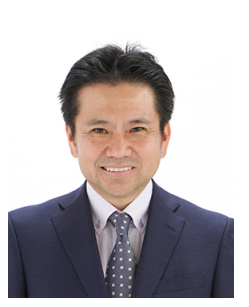

Takaya Yamazato was born in Okinawa, Japan, in 1964. He is a professor at the Institute of Liberal Arts and Sciences, Nagoya University, Japan. He received a Ph.D. degree from the Department of Electrical Engineering, Keio University, Yokohama, Japan, in 1993. From 1993 to 1998, he was an assistant professor at the Department of Information Electronics, Nagoya University, Japan. From 1997 to 1998, he was a visiting researcher at the Research Group for RF Communications, University of Kaiserslautern. In 1998, he gave a half-day tutorial entitled "Introduction to CDMA ALOHA" at Globecom held in Sydney, Australia. Since then, he has been serving as a TPC member of Globecom and ICC. In 2006, he received the IEEE Communication Society's Best Tutorial Paper Award. He served as the co-chair of the Wireless Communication Symposia of ICC 2009 and is the co-chair of Selected Areas in Communication Symposia of ICC 2011. From 2008 to 2010, he served as the chair of the Satellite and Space Communication Technical Committee. In 2011, he gave a half-day tutorial entitled "Visible Light Communication" at ICC 2011 held in Kyoto, Japan. He served as the editor-in-chief of the Japanese section of IEICE transactions on communications from 2009 to 2011. From 2016, he serves as the director of the Asia/Pacific Region (AP), IEEE Communication Society. His research interests include visible light communication, intelligent transport system, stochastic resonance, and open educational resources. (Email: yamazato@nagoya-u.jp) 